\title{
ENFERMEDAD DE CARRIÓN GRAVE COMPLICADA CON LEPTOSPIROSIS AGUDA: REPORTE DE UN CASO
}

\author{
Anderson Huerta ${ }^{1, a}$, Marcia Salas ${ }^{1,2, b}$, Jorge Yarinsueca ${ }^{1,2, c}$, Ciro Maguiña ${ }^{3, d}$
}

\begin{abstract}
RESUMEN
Se reporta el caso de un adulto varón de 56 años, procedente de Sihuas (Ancash) que acude al Hospital Nacional Dos de Mayo con cuadro clínico caracterizado por un síndrome ictérico febril anemizante, en cuya evolución tórpida de solo tres días de hospitalizado presenta anemia severa ( $\mathrm{Hb}: 11,8$ a 6,7 g/dL) así como mialgias generalizadas asociadas a un empeoramiento brusco de la ictericia correlacionada con elevación de bilirrubinas (BT: 3,2 a 19,6 mg/dL a predominio directo) y deterioro general del paciente; por ello, se realizó la investigación microbiológica donde se encontró frotis y cultivo positivo para Bartonella bacilliformis, posteriormente, la serología (microaglutinación) resultó positiva para Lesptospira serovar Pomona. El paciente recibe tratamiento antibiótico específico (ceftriaxona y ciprofloxacino), se le transfunde dos paquetes globulares, mejora clínicamente y es dado de alta.
\end{abstract}

Palabras clave: Enfermedad de Carrión; Bartonella bacilliformis; Leptospirosis (fuente: DeCS BIREME).

\section{CARRIÓN`S DISEASE COMPLICATED WITH SEVERE ACUTE LEPTOSPIROSIS: A CASE REPORT}

\begin{abstract}
The case of an adult, 56 year old male is reported, from Sihuas (Ancash) who presented at Hospital Nacional Dos de Mayo with fever, jaundice and anemia. In three days of hospitalization, he developed severe anemia (Hb: 11.8 to $6.7 \mathrm{~g} / \mathrm{dL}$ ) and generalized myalgias associated with a sudden worsening jaundice correlated with elevated bilirubin (TB 3.2 to $19.6 \mathrm{mg} / \mathrm{dL}$ direct dominance) and general deterioration. Microbiological smear and culture were positive for Bartonella bacilliformis. Subsequent serology (microagglutination) was positive for Lesptospira serovar Pomona. The patient was given specific antibiotic therapy (ceftriaxone and ciprofloxacin), transfused two units of packed red blood cells, improved clinically and was discharged.
\end{abstract}

Key word: Carrion's disease; Bartonella bacilliformis; Leptospirosis (source: MeSH NLM).

\section{INTRODUCCIÓN}

La enfermedad de Carrión es una enfermedad infecciosa asociada de manera especial a la historia del Hospital Nacional Dos de Mayo; el 27 de agosto de 1885, Daniel Alcides Carrión se inocula la sangre de una verruga peruana (localizada en la ceja derecha) de un paciente hospitalizado en la sala Las Mercedes de dicho nosocomio, sufre la enfermedad aguda y fallece el 5 de octubre de 1885; con ello demostró que era posible la transmisión de la enfermedad de Carrión y el carácter bifásico de la misma (fiebre de la Oroya y verruga peruana); convirtiéndose así en mártir de la medicina peruana ${ }^{(1,2)}$.

La enfermedad de Carrión es una infección metaxénica presente en el Perú, causada por la Bartonella bacilliformis, patógeno gramnegativo, polimórfico y flagelado; transmitido por mosquitos flebótomos del género Lutzomyia, particularmente la especie verrucarum ${ }^{(1,2,3)}$. Esta infección se desarrolla en valles costeros, regiones interandinas y selva alta del Perú, además de Ecuador y Colombia ${ }^{(3)}$. Existen varias formas clínicas: la fase aguda (fiebre de la Oroya), la fase

\footnotetext{
Facultad de Medicina, Universidad Nacional Mayor de San Marcos. Lima, Perú.

Hospital Nacional Dos de Mayo. Lima, Perú.

Instituto de Medicina Tropical “Alexander von Humboldt”, Universidad Peruana Cayetano Heredia. Lima, Perú.

Médico cirujano; ${ }^{b}$ Médica internista. Magíster en Docencia e Investigación en Salud; ${ }^{c}$ Médico internista; ${ }^{\mathrm{d}}$ Médico infectólogo tropicalista.

Recibido: 11-04-14 Aprobado: 28-05-14
}

Citar como: Huerta A, Salas M, Yarinsueca J, Maguiña C. Enfermedad de Carrión grave complicada con leptospirosis aguda: reporte de un caso. Rev Peru Med Exp Salud Publica. 2014;31(2):380-4. 
eruptiva o crónica (verruga peruana) y la bacteriemia asintomática ${ }^{(2,4)}$.

La leptospirosis es una enfermedad zoonótica de amplia distribución geográfica que afecta a animales silvestres, domésticos y, accidentalmente, al hombre, es causada por una bacteria espiroqueta del género Leptospira, el cual es un microorganismo helicoidal, aeróbico obligatorio ${ }^{(5,6)}$. Tiene distribución mundial, pero es más frecuente en áreas tropicales, como la selva peruana, donde encuentra las condiciones para su transmisión ${ }^{(5-7)}$. Actualmente esta enfermedad es de poca sospecha diagnóstica, al igual que sus formas graves, a pesar de estar o no en zonas endémicas de nuestro país ${ }^{(7)}$. Clínicamente presenta el síndrome febril indiferenciado, ictérico y hemorrágico ${ }^{(5,6)}$.

En el Perú, la enfermedad de Carrión es considerada una enfermedad reemergente y un problema de salud pública ${ }^{(8,9)}$. Están descritas las complicaciones presentes en la fase aguda (anemizante) de la enfermedad de Carrión, de las cuales el $36,5 \%$ representa a las infecciones sobreagregadas, entre ellas la leptospirosis ${ }^{(4,8)}$. Se reporta el caso de un paciente con diagnóstico de enfermedad de Carrión grave complicada con una superinfección por Leptospira serovar Pomona, acontecido en el Hospital Nacional Dos de Mayo de Lima, Perú.

\section{REPORTE DE CASO}

Paciente varón de 56 años, natural de Ayacucho, procedente de Sihuas (Ancash), residente en el lugar desde los 14 años, de ocupación soldador, también desde los 14 años, su centro laboral se encuentra próximo a un río, lugar donde lava sus herramientas. Desde hace 5 años no ha viajado a otros lugares fuera de Ancash. Ingresa al Servicio de Emergencia del Hospital Nacional Dos de Mayo el 03/05/2013, y luego a la sala de medicina Santo Toribio, con un tiempo de enfermedad de 20 días, caracterizado por sensación de alza térmica no cuantificada a predominio nocturno que cedía parcialmente con toma de antipiréticos, y malestar general. Tres días antes del ingreso presentó náuseas, vómitos, palidez e ictericia. Por persistencia del cuadro decide viajar directamente a Lima para su atención. Niega tos, diarreas, y dolor abdominal. Antecedentes: enfermó de tuberculosis pulmonar (hace 20 años) sin tratamiento. Antecedentes familiares: tiene cuatro hijos, dos mujeres y dos varones (todos con TBC pulmonar hace 15 años con tratamiento completo).

Al examen físico sus funciones vitales fueron: PA: 90/50 mmHg. FC: 120 latidos/min. FR: 28 respiraciones/ minuto. T: $38,5^{\circ} \mathrm{C}$ (oral). Paciente en regular estado general, mal estado de hidratación, regular estado de nutrición, orientado, facies dolorosa (refiere dolor intenso en hombro izquierdo), luce sudoroso, quejumbroso, ventilando espontáneamente. Piel: caliente, poco hidratada, ictericia en escleras y piel $(++/+++)$, palidez $(++/+++)$. Linfáticos: adenopatías retrocervicales bilaterales de aprox. 0,5-1 cm, móviles, blandos, no dolorosos, adenopatías inguinales de +/$1,5 \mathrm{~cm}$ de diámetro de similares características a las anteriores. Aparato respiratorio: murmullo vesicular pasa bien en ambos hemitórax, respiración ruda. Aparato cardiovascular: ruidos cardiacos rítmicos, poco intensos, taquicárdicos, no soplos. Abdomen: blando, depresible, dolor a palpación profunda difuso, a predominio de hipocondrio derecho, hígado a $6 \mathrm{~cm}$ debajo de reborde costal derecho, spam hepático de $13 \mathrm{~cm}$; bazo no palpable, signo de Murphy positivo. Sistema neurológico: Glasgow de 15 puntos. Resto del examen físico sin alteraciones.

Dentro de los exámenes de laboratorio, al ingreso, $\mathrm{Hb}$ : 11,8 mg/dL, grupo: O +; glucosa: $102 \mathrm{mg} / \mathrm{dL}$; reticulocitos: $3 \%$, test de Coombs directo negativo. Anticuerpos hepatitis $\mathrm{C}$ no reactivo. Antígeno de superficie hepatitis $B$ no reactivo. Perfil coagulación normal. Electrolitos seriados dentro de límites normales. En la radiografía de tórax se informó engrosamiento pleural apical derecho e imágenes reticulares en pulmón derecho a predominio de ápice (probablemente debido a secuela de proceso inflamatorio previo); posteriormente, las baciloscopías para tuberculosis pulmonar en esputo seriado dieron resultados negativos en tres ocasiones.

En los tres primeros días de hospitalizado se observó deterioro progresivo del estado general, mialgias generalizadas exacerbadas a la palpación, severa palidez e ictericia que fue incrementando. En la Tabla 1 se evidencia caída marcada y persistente de los niveles de hemoglobina, llegando a nivel de anemia severa ( $\mathrm{Hb}: 6,7 \mathrm{~g} / \mathrm{dL}$ ), duplicación de los niveles leucocitarios en solo un día (leucocitosis severa) con desviación izquierda y proteína $\mathrm{C}$ reactiva elevada. Dado que el paciente presentó fiebre, palidez marcada e ictericia, se sospecha un proceso infeccioso general y se plantean los diagnósticos diferenciales de enfermedad de Carrión (procedía de Ancash), hepatitis viral y colangitis aguda, por ello, se envían muestras de sangre a los laboratorios del Instituto de Medicina Tropical "Daniel Alcides Carrión" de la UNMSM y del Instituto Nacional de Salud, que luego resultaron ambas positivas para Bartonella bacilliformis en coloración Giemsa (Figura 1 ); posteriormente se reciben los resultados de cultivo positivos para dicho agente. Dada la evolución tórpida y la persistencia de la fiebre y el deterioro clínico del paciente, se investigó infecciones sobreagregadas con 
Tabla 1. Características laboratoriales seriadas de los hemogramas completos y proteína $\mathrm{C}$ reactiva

\begin{tabular}{|c|c|c|c|c|c|c|}
\hline Examen auxiliar & Ingreso & Día 2 & Día 3 & Día 4 & Día 5 & Día 12 \\
\hline Hemoglobina & 11,8 & 8,5 & 6,7 & - & 7,4 & 8,3 \\
\hline Leucocitos & 15690 & 30150 & 19240 & - & 11320 & 8870 \\
\hline Abastonados & $8 \%$ & $8 \%$ & $9 \%$ & - & $8 \%$ & $0 \%$ \\
\hline Segmentados & $72 \%$ & $80 \%$ & $63 \%$ & - & $55 \%$ & $64 \%$ \\
\hline Eosinófilos & $1 \%$ & $0 \%$ & $0 \%$ & - & $0 \%$ & $3 \%$ \\
\hline Linfocitos & $11 \%$ & $8 \%$ & $4 \%$ & - & $20 \%$ & $24 \%$ \\
\hline Plaquetas & 87000 & 54000 & 123000 & - & 142000 & 531000 \\
\hline Observación & Granulaciones tóxicas & - & Granulaciones tóxicas & - & - & - \\
\hline PCR & - & 241 & - & 229 & - & 39 \\
\hline
\end{tabular}

las siguientes pruebas: aglutinaciones (tífico, paratífico y brucela); hemocultivos repetidos en dos ocasiones, urocultivo y mielocultivo, todos resultaron negativos, pero la microaglutinación resultó positiva en título 1/600 para Leptospira pomona. Con estos resultados se llegó a la conclusión que el paciente presentaba enfermedad de Carrión grave complicada con leptospirosis aguda.

En la evolución se evidenció mejoría tanto en patrones clínicos como bioquímicos (Tabla 1) normalizándose gradualmente los niveles de urea y creatinina (Tabla 2). Se transfundió dos paquetes globulares y se instauró el tratamiento antibiótico específico (ceftriaxona y ciprofloxacino) según norma técnica vigente ${ }^{(3)}$, y luego de 10 días, es dado de alta del servicio.

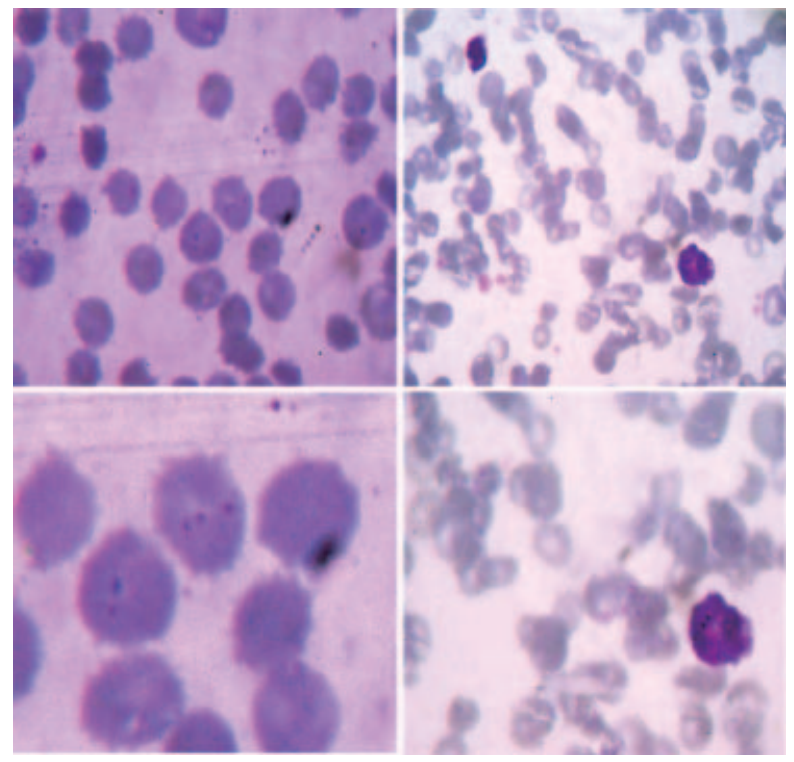

Figura 1. Lámina periférica sanguínea con imágenes cocobacilares intraeritrocitarias correspondientes a Bartonella bacilliformis vistas en el laboratorio de (editado para mantener confidencialidad). Coloración Giemsa (aumento $2 X$ y $3 X$ )
El paciente expresó de manera verbal su autorización para emplear sus datos clínicos en la elaboración de esta comunicación.

\section{DISCUSIÓN}

En el período de 1945 a 1994, el Ministerio de Salud reportó que la mayoría de casos diagnosticados de enfermedad de Carrión procedían de Ancash (83,4\%). Desde 1993 se evidenció aumento de casos en zonas consideradas no endémicas. En el año 2001, el 52,6\% del total de casos a nivel nacional procedieron de Ancash, y el resto de otras regiones de Perú ${ }^{(2,5,8)}$. En el período de 1994 a 2004 se confirmaron casos de leptospirosis en 18 de las 24 regiones del Perú. La región que más casos confirmados reportó fue Loreto $(21,6 \%)$, seguida de Cusco $(14,8 \%)$; mientras que las regiones que reportaron menos casos fueron Ancash, Ayacucho, Amazonas $(<2 \%)^{(6,7)}$.

En la evolución del perfil bioquímico hepático (ver Tabla 3), se aprecia el incremento brusco y sostenido del nivel de bilirrubinas totales (de 3,2 a 19,6 mg/dL) a predominio directo, posiblemente relacionado con daño hepatocelular o séptico. La clínica desfavorable al segundo día de hospitalización, y la evidencia de tríada

Tabla 2. Características laboratoriales seriadas de los perfiles hepáticos

\begin{tabular}{lcccc}
\hline Examen auxiliar & Ingreso & Día 3 & Día 4 & Día 12 \\
\hline Proteina total & 5,64 & - & 4,85 & 6,07 \\
Albumina & 2,40 & 1,67 & 1,56 & 2,36 \\
Bilirubina total & 3,2 & 19,6 & 15,8 & 2,8 \\
Bilirubina directa & 1,3 & 18,4 & 14,7 & 2,2 \\
Bilirubina indirecta & 1,9 & 1,2 & 1,1 & 0,6 \\
Fosfatasa alcalina & 317 & 149 & 123 & 238 \\
TGO & 56 & 57 & 39 & 32 \\
TGP & 92 & 68 & 54 & 54 \\
\hline
\end{tabular}


Tabla 3. Características laboratoriales seriadas de marcadores de función renal y exámenes de orina

\begin{tabular}{lcccc}
\hline $\begin{array}{c}\text { Examen } \\
\text { auxiliar }\end{array}$ & Ingreso & Día 6 & Día 8 & Día 11 \\
\hline Úrea & - & 55 & 31 & 23 \\
Creatinina & - & 1,42 & 1,21 & 0,9 \\
Orina & & & & \\
Leucocitos & $0-2 / c$ & & & $1-2 / c$ \\
Proteínas & ++ & & & - \\
Bilirrubina & + & - & - & - \\
Sangre & + & & & - \\
\hline
\end{tabular}

de Charcot (fiebre, ictericia y dolor a la palpación en hipocondrio derecho) hizo sospechar colangitis aguda condicionada por coledocolitiasis, por lo que se inició tratamiento empírico con ceftriaxona y metronidazol. Posteriormente, dos ecografías abdominales permitieron descartar este diagnóstico. Se empezó el tratamiento específico empírico para enfermedad de Carrión en fase aguda, continuando con la aplicación de ceftriaxona y agregando ciprofloxacino; al ser confirmada la infección se continuó el tratamiento ${ }^{(3)}$.

Debido al deterioro clínico y laboratorial se sospechó de alguna enfermedad infecciosa sobreagregada por lo que se inició el proceso diagnóstico descrito previamente, y se evidenció títulos de aglutinación de 1/600 para Leptospira pomona, con la sospecha que adquirió la infección al lavar sus herramientas de trabajo en un río cercano a su centro laboral ${ }^{6,7)}$

La enfermedad de Carrión, causada por la Bartonella bacilliformis, es descrita por la ciencia médica como un estado de depresión transitoria de la inmunidad celular durante la fase aguda anemizante, se puede presentar leve linfopenia y disminución significativa de linfocitos T. También se ha observado disminución de los niveles de linfocitos CD4 y elevación de los niveles de linfocitos CD8, ello explica la alta frecuencia de infecciones oportunistas agregadas ${ }^{(2,4,9)}$.

Las complicaciones de la enfermedad de Carrión se presentan con frecuencia en la etapa aguda anemizante y de inmunosupresión. De estas se reportan dos tipos: las infecciosas y las no infecciosas; en cada una de ellas la mortalidad puede a llegar a ser alta con tasa de letalidad de 16 y $9 \%$ respectivamente ${ }^{(2,4,9)}$. Dentro de las complicaciones infecciosas que suponen el $36,7 \%$, se encuentran las bacterianas, de las cuales se han hallado la presencia de infecciones oportunistas como salmonela no tífica (Salmonella typhimurium, S. enteritidis.), Salmonella typhi, reactivación de toxoplasmosis, histoplasmosis diseminada, sepsis (Staphylococcus aureus, Enterobacter spp., Shigella dysenteriae, Pseudomonas aeruginosa, Acinetobacter); neumocystosis, malaria por $P$. vivax, tuberculosis reactivada, tifus, hepatitis $\mathrm{B}$ y leptospirosis $(2,4,10,11)$. La anemia es de tipo hemolítico y es rápida y progresiva. Por ello se encuentran bajos niveles de hemoglobina e incremento de bilirrubina indirecta, pero en la fase aguda de la enfermedad también existe compromiso hepático en grado variable, por ello se puede encontrar predominio de bilirrubina directa, como ocurrió en este caso ${ }^{(10)}$.

La leptospirosis puede presentarse como varios síndromes clínicos, clásicamente se ha descrito una fase febril anictérica y la enfermedad de Weill (fase febril ictérica grave). Maguiña et al. han descrito la ictericia de manera importante en la fase aguda de la enfermedad, en este paciente la ictericia inicial llevó de manera inicial a descartar un problema de vías biliares (coledocolitiasis o colangitis aguda), la cual se descartó y llevó al planteamiento de una enfermedad bacteriana infecciosa $y$, dada la procedencia de Ancash, se pensó en la enfermedad de Carrión, lo que se confirmó, pero por la evolución tórpida del caso, cobró importancia la leptospirosis como infección oportunista ${ }^{(10)}$. Esta complicación secundaria en la enfermedad de Carrión ha sido descrita en pocos artículos de la literatura médica. En 1999, Nuñez et al. describen casos de leptospirosis como complicación de la enfermedad de Carrión, con cuadros purpúricos y presentación clínica inusual en Quillabamba, incluyendo cuadros de sepsis en el 25\% (5/16) de los pacientes encontrados, esta información es recogida por Pachas et al en el $2001^{(2,14)}$. En el 2007, Pachas et al reportaron que $54 \%(29 / 53)$ de pacientes con enfermedad de Carrión en fase aguda presentaron superinfección asociada a la leptospirosis ${ }^{(15)}$.

Agradecimientos: a los doctores Marcos Ñavincopa y Yuri García, médicos infectólogos del Hospital Nacional Dos de Mayo; y a la médica residente, Dra. Maribel Condori, quienes con su preocupación y valiosos aportes en el diagnóstico y tratamiento, hicieron posible un oportuno y adecuado manejo conjunto de nuestro paciente.

Fuentes de financiamiento: autofinanciada.

Conflictos de interés: los autores declaran no tener conflictos de interés. 


\section{REFERENCIAS BIBLIOGRÁFICAS}

1. Sanchez N, Ugarte-Gil C, Solorzano N, Maguiña C, Pachas P, Blazes D, et al. Bartonella bacilliformis: A Systematic Review of the Literature to Guide the Research Agenda for Elimination. PLoS Negl Trop Dis 2012;6(10):e1819. doi:10.1371/journal.pntd.0001819

2. Perú, Ministerio de Salud. Enfermedad de Carrión (Bartonelosis) en el Perú. Lima: MINSA; 2001.

3. Tarazona A, Maguiña C, López de Guimaraes D, Montoya M, Pachas P. Terapia antibiótica para manejo de la bartonelosis o enfermedad de Carrión en el Perú. Rev Peru Med Exp Salud Publica. 2006;23(3):180-200.

4. Maguiña Vargas C, Ugarte-Gil C, Breña Chávez P, Ordaya Espinoza E, Ventosilla López P, Huarcaya Castilla $\mathrm{E}$, et al. Actualización de la enfermedad de Carrión. Rev Med Hered. 2008;19(1):36-41.

5. Cruz-Vilchez JE, Vargas-Cruz MA. Bartonelosis aguda complicada. Presentación de 44 casos en Huancabamba, Piura. Rev Soc Per Med Inter. 2003;16(4):5-10.

6. Céspedes M, Balda L, Gonzales D, Tapia R. Situación de la leptospirosis en el Perú 1994 - 2004. Rev Peru Med Exp Salud Publica. 2006;23(1):56-96.

7. Perú, Ministerio de Salud. Norma técnica de salud para la atención integral de la persona afectada con leptospirosis. Lima: MINSA; 2006.

8. Pachas P. Epidemiología de la bartonelosis en el Perú. Módulos Técnicos-Serie Documentos Monográficos. Lima: Instituto Nacional de Salud; 2000.

9. Maguiña Vargas CP, Peña Collantes RB, Ponce Álvarez MC, Quispe-Chullo TF, Ventosilla López PR. Manifestaciones clínicas y complicaciones de la fase aguda de bartonelosis o Fiebre de la Oroya en pacientes atendidos en el Hospital Nacional Cayetano Heredia. Rev Med Hered. 2008;19(3):87-95.

10. Maguina C, Garcia PJ, Gotuzzo E, Cordero L, Spach DH. Bartonellosis (Carrion's disease) in the modern era. Clin Infect Dis. 2001 Sep 15;33(6):772-9.

11. Maguiña C, Gotuzzo E. Bartonellosis. New and old. Infect Dis Clin North Am. 2000 Mar;14(1):1-22, vii.

12. Gotuzzo Herencia E. Enfermedades emergentes y reemergentes en el Perú. Rev Med Hered. 2002;13(2):37-9.
13. Segura ER, Ganoza CA, Campos K, Ricaldi JN, Torres S, Silva H, et al. Clinical spectrum of pulmonary involvement in leptospirosis in a region of endemicity, with quantification of leptospiral burden. Clin Infect Dis. 2005 Feb 1;40(3):343-51.

14. Núñez G, Canales J, Cjuno R, Grajeda P, González C. Brote de bartonelosis en la provincia de La Convención-Cusco 1998. Situa. 1999;(7):20-4.

15. Pachas P, Aranda M, Troyes L, Matos Arias J, Padilla C, Ventura G, et al. Superinfections associated with human bartonellosis among in patiens in the northern forest of Peru [abstracts]. ASTMH $56^{\text {th }}$ annual meeting. Pennsylvania, EEUU; November 4-8, 2007.

Correspondencia: Anderson Valentin
Huerta Obando
Dirección: AAHH 31 de diciembre Mz.L,
Lt.7. Independencia
Teléfono: 954704753
Correo electrónico: huertaandy@hotmail.com
Huerta Obando

Dirección: AAHH 31 de diciembre Mz. L, Teléfono: 954704753

Correoelectrónico:huertaandy@hotmail.com

\section{Consulte las ediciones anteriores de la} Revista Peruana de Medicina Experimental y Salud Pública en

\section{www.scielosp.org}

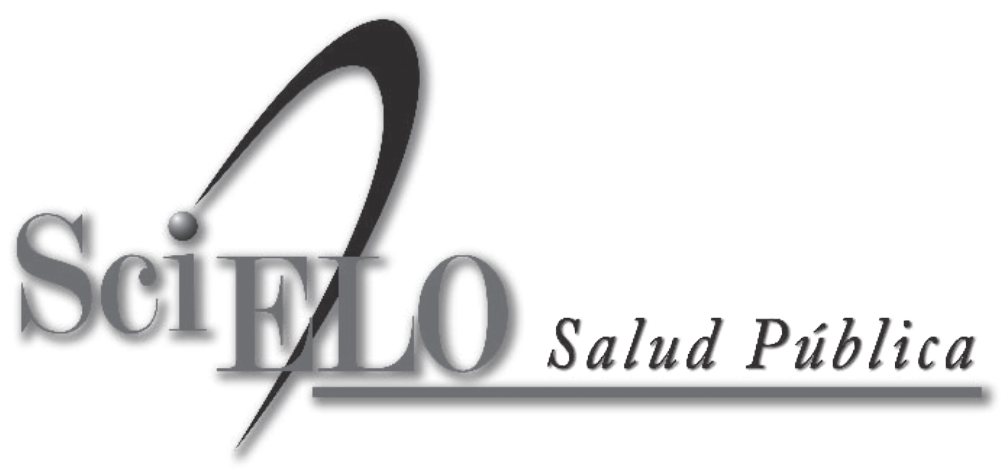

